total lymph drainage volume was significantly lower in group 1 than in group $3(663 \pm 292 \mathrm{ml}$ vs $1,040 \pm 739 \mathrm{ml} ; P=0.03$ ).

The authors conclude that heparin should not be routinely administered to low-risk living donor kidney transplant recipients.

Original article Osman Y et al. (2007) Necessity of routine postoperative heparinization in non-risky live-donor renal transplantation: results of a prospective randomized trial. Urology 69: 647-651

\section{Good outcomes with ABO- incompatible transplantation: report from three centers}

A new protocol for $\mathrm{ABO}$-incompatible kidney transplantation was proposed in 2001 after preliminary success at a transplant center in Stockholm, Sweden. Now, Tydén and coauthors report on the successful implementation of the protocol at this center and at two other institutions, in Uppsala, Sweden, and Freiberg, Germany. No differences were found between the outcomes of 60 consecutive patients who received ABOincompatible living-donor transplants under the new protocol and those of 274 patients

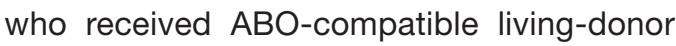
transplants during the same period.

The new protocol comprises the following: preoperative antigen-specific immunoadsorption to remove existing $\mathrm{ABO}$ antibodies; single-dose rituximab $\left(375 \mathrm{mg} / \mathrm{m}^{2} 4\right.$ weeks before immunoadsorption) and intravenous immunoglobulin $(0.5 \mathrm{~g} / \mathrm{kg}$ before transplantation) to prevent antibody rebound; and conventional immunosuppression with tacrolimus, mycophenolate mofetil and prednisolone, started about a week before immunoadsorption. After a mean follow-up of 17.5 months (range 2-61 months), ABO-incompatible graft survival was $97 \%$, and the mean serum creatinine level of recipients was $127 \mu \mathrm{mol} / \mathrm{l}(1.44 \mathrm{mg} / \mathrm{dl})$; corresponding figures for $\mathrm{ABO}$-compatible grafts were $95 \%$ and $133 \mu \mathrm{mol} / /(1.50 \mathrm{mg} / \mathrm{dl})$. Of the two graft losses observed with the new protocol, one was attributed to noncompliance with the immunosuppressive regimen, and one was the result of patient death from Clostridium $\mathrm{sp}$. infection at 4 months. Patient survival was $98 \%$ in each group.

No late rebound of antibodies or humoral rejection was observed under the new protocol. Substantial institutional variation in antibody titers led to a marked difference in the number of immunoadsorption sessions required, indicating a need for a standardized fluorescence-activated cell-sorting technique.

Original article Tydén G et al. (2007) Implementation of a protocol for $\mathrm{ABO}$-incompatible kidney transplantation-a three-center experience with 60 consecutive transplantations. Transplantation 83: 1153-1155

\section{Good long-term outcome with non-heartbeating kidney transplantation}

A meta-analysis of studies that compared transplantation of kidneys from non-heartbeating (NHB) and heartbeating (HB) donors has been conducted. The results show that, although use of kidneys from NHB donors is associated with an increased incidence of adverse events in the short term, longer-term outcomes are comparable with those from HB donation.

The analysis included 18 retrospective studies published during the period 1992-2005, totaling 1,858 NHB donations (also known as 'donation after cardiac death' and 'asystolic donation'), and $112,223 \mathrm{HB}$ donations from cadaveric donors. NHB kidneys had a significantly longer warm ischemia time than did HB kidneys (by $24 \mathrm{~min}$; $P<0.001)$. Cold ischemia times did not differ markedly. Transplantation of organs from NHB donors was associated with higher incidences of primary nonfunction and delayed function $(P<0.001$ for both), but not of acute rejection. Recipients of grafts from NHB donors also had a longer hospital stay (by 4.5 days; $P<0.001$ ).

The graft survival advantage observed in the HB group decreased as the studies progressed, however, and was nonsignificant at 10 years following transplantation. Interestingly, patient survival was higher in the NHB group during the initial postoperative period, but the difference was nonsignificant at 6 months and at all subsequent analysis points up to the end of follow-up (6 years).

This study indicates that NHB kidney donation could help to redress the current shortage of kidney donors; the authors believe that the elevated early risks of primary nonfunction and delayed graft function are offset by the promise of good long-term outcome.

Original article Kokkinos C et al. (2007) Outcome of kidney transplantation from nonheart-beating versus heart-beating cadaveric donors. Transplantation 83: 1193-1199 